\title{
Resolving the Crab pulsar wind nebula at teraelectronvolt energies
}

\author{
H.E.S.S. Collaboration: H. Abdalla ${ }^{1}$, F. Aharonian ${ }^{2,3,4}$, F. Ait Benkhali ${ }^{2}$, E.O. Angüner ${ }^{5}$, M. Arakawa ${ }^{6}$, C. Arcaro ${ }^{1}$, C. Armand ${ }^{7}$, M. Backes ${ }^{8}, 1$, \\ M. Barnard ${ }^{1}$, Y. Becherini ${ }^{9}$, J. Becker Tjus ${ }^{10}$, D. Berge ${ }^{11}$, K. Bernlöhr ${ }^{2}$, R. Blackwell ${ }^{12}$, M. Böttcher ${ }^{1}$, C. Boisson ${ }^{13}$, J. Bolmont ${ }^{14}$, S. Bonnefoy ${ }^{11}$,

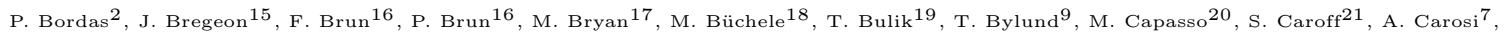 \\ S. Casanova ${ }^{22,2}$, M. Cerruti ${ }^{14,23}$, N. Chakraborty ${ }^{2}$, T. Chand ${ }^{1}$, S. Chandra ${ }^{1}$, R.C.G. Chaves ${ }^{15,24}$, A. Chen ${ }^{25}$, S. Colafrancesco ${ }^{25,45}$, B. Condon ${ }^{26}$, \\ I.D. Davids ${ }^{8}$, C. Deil ${ }^{2}$, J. Devin ${ }^{15}$, P. deWilt ${ }^{12}$, L. Dirson ${ }^{27}$, A. Djannati-Ataï ${ }^{28}$, A. Dmytriiev ${ }^{13}$, A. Donath ${ }^{2}$, V. Doroshenko ${ }^{20}$, L.O'C. Drury ${ }^{3}$, \\ J. Dyks ${ }^{29}$, K. Egberts ${ }^{30}$, G. Emery ${ }^{14}$, J.-P. Ernenwein ${ }^{5}$, S. Eschbach ${ }^{18}$, S. Fegan ${ }^{21}$, A. Fiasson ${ }^{7}$, G. Fontaine ${ }^{21}$, S. Funk ${ }^{18}$, M. Füßling ${ }^{11}$, S. Gabici ${ }^{28}$, \\ Y.A. Gallant ${ }^{15}$, F. Gaté 7 , G. Giavitto ${ }^{11}$, D. Glawion ${ }^{31}$, J.F. Glicenstein ${ }^{16}$, D. Gottschall ${ }^{20}$, M.-H. Grondin ${ }^{26}$, J. Hahn ${ }^{2}$, M. Haupt ${ }^{11}$, G. Heinzelmann ${ }^{27}$,

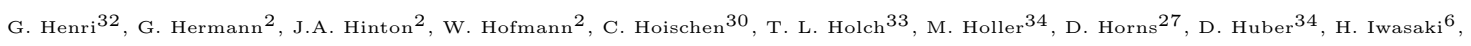 \\ A. Jacholkowska ${ }^{14,45}$, M. Jamrozy ${ }^{35}$, D. Jankowsky ${ }^{18}$, F. Jankowsky ${ }^{31}$, L. Jouvin ${ }^{28}$, I. Jung-Richardt ${ }^{18}$, M.A. Kastendieck ${ }^{27}$, K. Katarzyński ${ }^{36}$, \\ M. Katsuragawa ${ }^{37}$, U. Katz ${ }^{18}$, D. Khangulyan ${ }^{6}$, B. Khélifi ${ }^{28}$, J. King ${ }^{31}$, S. Klepser ${ }^{11}$, W. Kluźniak ${ }^{29}$, Nu. Komin ${ }^{25}$, K. Kosack ${ }^{16}$, M. Kraus ${ }^{18}$, \\ G. Lamanna ${ }^{7}$, J. Lau ${ }^{12}$, J. Lefaucheur ${ }^{13}$, A. Lemière ${ }^{28}$, M. Lemoine-Goumard ${ }^{26}$, J.-P. Lenain ${ }^{14}$, E. Leser ${ }^{30}$, T. Lohse ${ }^{33}$, R. López-Coto ${ }^{2}$, I. Lypova ${ }^{11}$, \\ D. Malyshev ${ }^{20}$, V. Marandon ${ }^{2}$, A. Marcowith ${ }^{15}$, C. Mariaud ${ }^{21}$, G. Martí-Devesa ${ }^{34}$, R. Marx ${ }^{2}$, G. Maurin ${ }^{7}$, P.J. Meintjes ${ }^{38}$, A.M.W. Mitchell ${ }^{2,39}$, \\ R. Moderski ${ }^{29}$, M. Mohamed ${ }^{31}$, L. Mohrmann ${ }^{18}$, C. Moore ${ }^{40}$, E. Moulin ${ }^{16}$, T. Murach ${ }^{11}$, S. Nakashima ${ }^{41}$, M. de Naurois ${ }^{21}$, H. Ndiyavala ${ }^{1}$, \\ F. Niederwanger ${ }^{34}$, J. Niemiec ${ }^{22}$, L. Oakes ${ }^{33}$, P. O'Brien ${ }^{40}$, H. Odaka ${ }^{42}$, S. Ohm ${ }^{11}$, M. Ostrowski ${ }^{35}$, I. Oya ${ }^{11}$, M. Panter ${ }^{2}$, R.D. Parsons ${ }^{2}$, C. Perennes ${ }^{14}$,

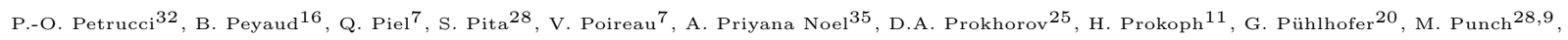 \\ A. Quirrenbach ${ }^{31}$, S. Raab ${ }^{18}$, R. Rauth ${ }^{34}$, A. Reimer ${ }^{34}$, O. Reimer ${ }^{34}$, M. Renaud ${ }^{15}$, F. Rieger ${ }^{2}$, L. Rinchiuso ${ }^{16}$, C. Romoli ${ }^{2}$, G. Rowell ${ }^{12}$, B. Rudak ${ }^{29}$, \\ E. Ruiz-Velasco ${ }^{2}$, V. Sahakian ${ }^{43,4}$, S. Saito ${ }^{6}$, D.A. Sanchez ${ }^{7}$, A. Santangelo ${ }^{20}$, M. Sasaki ${ }^{18}$, R. Schlickeiser ${ }^{10}$, F. Schüssler ${ }^{16}$, A. Schulz ${ }^{11}$, H. Schutte ${ }^{1}$, \\ U. Schwanke ${ }^{33}$, S. Schwemmer ${ }^{31}$, M. Seglar-Arroyo ${ }^{16}$, M. Senniappan ${ }^{9}$, A.S. Seyffert ${ }^{1}$, N. Shafi ${ }^{25}$, I. Shilon ${ }^{18}$, K. Shiningayamwe ${ }^{8}$, R. Simoni ${ }^{17}$,

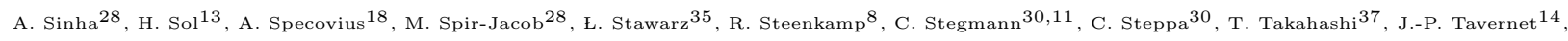

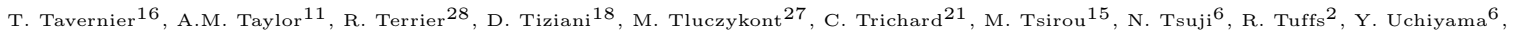 \\ D.J. van der Walt ${ }^{1}$, C. van Eldik ${ }^{18}$, C. van Rensburg ${ }^{1}$, B. van Soelen ${ }^{38}$, G. Vasileiadis ${ }^{15}$, J. Veh ${ }^{18}$, C. Venter ${ }^{1}$, P. Vincent ${ }^{14}$, J. Vink ${ }^{17}$, F. Voisin ${ }^{12}$,

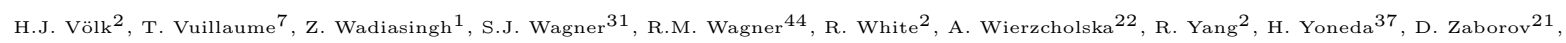 \\ M. Zacharias ${ }^{1}$, R. Zanin ${ }^{2}$, A.A. Zdziarski ${ }^{29}$, A. Zech ${ }^{13}$, A. Ziegler ${ }^{18}$, J. Zorn ${ }^{2}$, and N. Żywucka ${ }^{35}$ \\ ${ }^{1}$ Centre for Space Research, North-West University, Potchefstroom 2520, South Africa \\ 2 Max-Planck-Institut für Kernphysik, P.O. Box 103980, D 69029 Heidelberg, Germany \\ ${ }^{3}$ Dublin Institute for Advanced Studies, 31 Fitzwilliam Place, Dublin 2, Ireland \\ ${ }^{4}$ National Academy of Sciences of the Republic of Armenia, Marshall Baghramian Avenue, 24, 0019 Yerevan, Republic of Armenia \\ ${ }^{5}$ Aix Marseille Université, CNRS/IN2P3, CPPM, Marseille, France \\ ${ }^{6}$ Department of Physics, Rikkyo University, 3-34-1 Nishi-Ikebukuro, Toshima-ku, Tokyo 171-8501, Japan \\ ${ }^{7}$ Laboratoire d'Annecy de Physique des Particules, Univ. Grenoble Alpes, Univ. Savoie Mont Blanc, CNRS, LAPP, 74000 Annecy, France \\ ${ }^{8}$ University of Namibia, Department of Physics, Private Bag 13301, Windhoek, Namibia, 12010 \\ ${ }^{9}$ Department of Physics and Electrical Engineering, Linnaeus University, 35195 Växjö, Sweden \\ ${ }^{10}$ Institut für Theoretische Physik, Lehrstuhl IV: Weltraum und Astrophysik, Ruhr-Universität Bochum, D 44780 Bochum, Germany \\ ${ }^{11}$ DESY, D-15738 Zeuthen, Germany \\ 12 School of Physical Sciences, University of Adelaide, Adelaide 5005, Australia \\ ${ }^{13}$ LUTH, Observatoire de Paris, PSL Research University, CNRS, Université Paris Diderot, 5 Place Jules Janssen, 92190 Meudon, France \\ ${ }^{14}$ Sorbonne Université, Université Paris Diderot, Sorbonne Paris Cité, CNRS/IN2P3, Laboratoire de Physique Nucléaire et de Hautes Energies, LPNHE, 4 \\ Place Jussieu, F-75252 Paris, France \\ ${ }^{15}$ Laboratoire Univers et Particules de Montpellier, Université Montpellier, CNRS/IN2P3, CC 72, Place Eugène Bataillon, F-34095 Montpellier Cedex 5, \\ France
}




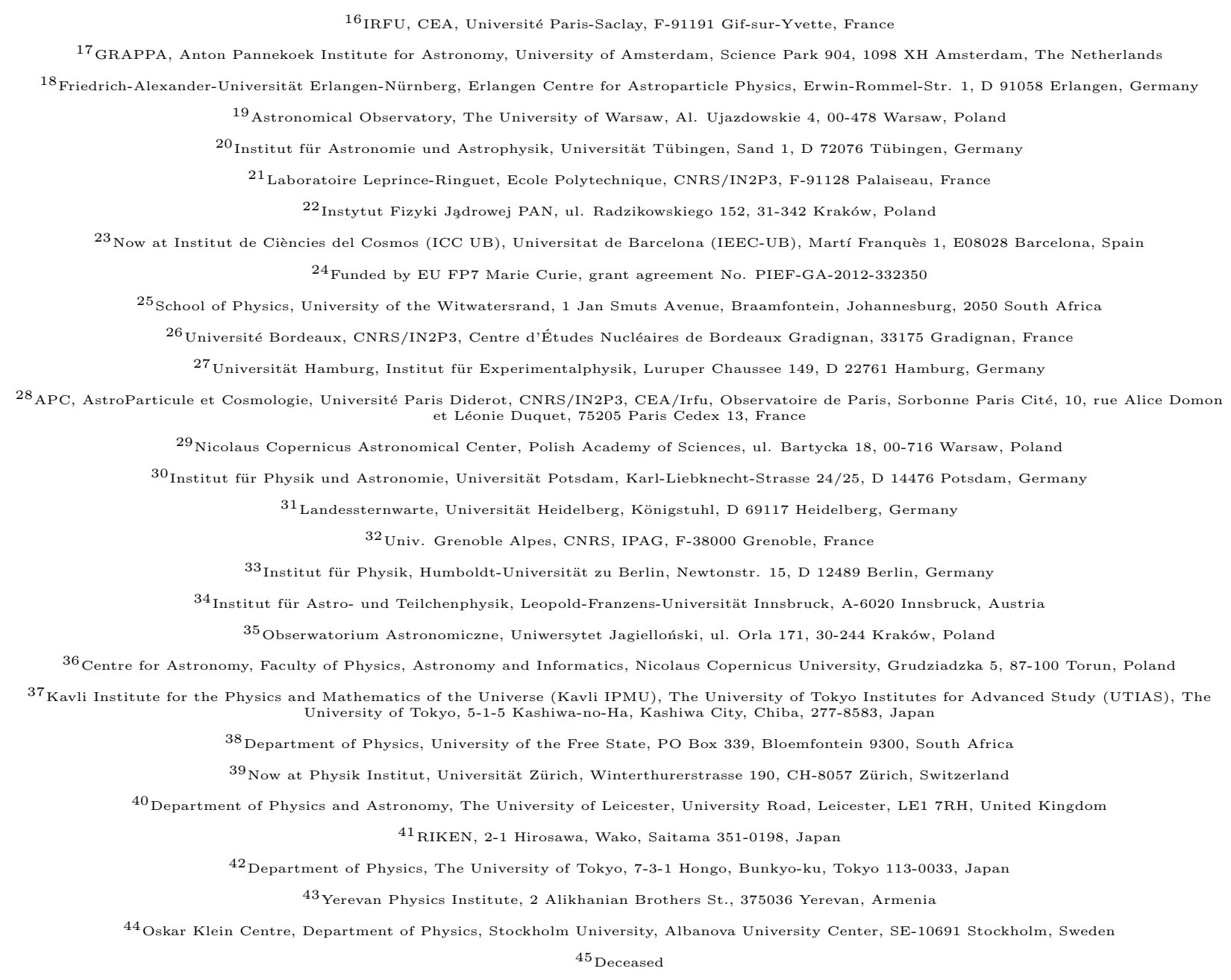

September 24, 2019

The Crab nebula is one of the most studied cosmic particle accelerators, shining brightly across the entire electromagnetic spectrum up to very high-energy gamma rays ${ }^{12}$. It is known from radio to gamma-ray observations that the nebula is powered by a pulsar, which converts most of its rotational energy losses into a highly relativistic outflow. This outflow powers a pulsar wind nebula (PWN), a region of up to 10 light-years across, filled with relativistic electrons and positrons. These particles emit synchrotron photons in the ambient magnetic field and produce very high-energy gamma rays by Compton up-scattering of ambient lowenergy photons. While the synchrotron morphology of the nebula is well established, it was up to now not known in which region the very high-energy gamma rays are emitted ${ }^{3}-8$. Here we report that the Crab nebula has an angular extension at gamma-ray energies of 52 arcseconds (assuming a Gaussian source width), significantly larger than at X-ray energies. This result 
closes a gap in the multi-wavelength coverage of the nebula, revealing the emission region of the highest energy gamma rays. These gamma rays are a new probe of a previously inaccessible electron and positron energy range. We find that simulations of the electromagnetic emission reproduce our new measurement, providing a non-trivial test of our understanding of particle acceleration in the Crab nebula.

The Crab pulsar's relativistic outflow is a cold, non-turbulent wind of charged particle pairs, electrons and positrons (commonly called electrons in the following). At a distance of 0.5 light-years from the pulsar, the wind is heated up by passing through a termination shock. Beyond this point, most of the electrons in the shocked wind have energies of $100-300 \mathrm{GeV}$ (gigaelectronvolts, $10^{9} \mathrm{eV}$ ), with maximum energies reaching up to $\mathrm{PeV}$ (petaelectronvolts, $10^{15} \mathrm{eV}$ ) energies. For a black body, such average thermal particle energies would be reached at extreme temperatures of $10^{15} \mathrm{~K}$, clearly demonstrating that this system is a non-thermal particle accelerator. The shocked wind continues propagating away from the pulsar, reaching at the present epoch out to distances of several light-years.

This part of the wind behind the termination shock is a region filled with radiating electrons producing radio to gamma-ray emission and is known as the Crab pulsar wind nebula (PWN, shown in Fig. 1). While the radio synchrotron emission of the Crab PWN was discovered in the 1960's (see e.g. ref. ${ }^{2}$ for a recent review), the Inverse Compton (IC) component of the PWN at GeV to TeV (teraelectronvolts, $10^{12} \mathrm{eV}$ ) photon energies was only discovered in 1989 with the Whipple telescope ${ }^{3}$. Today, the Crab nebula is the brightest steady source of $\mathrm{TeV}$ gamma rays in the sky and is the standard candle of gamma-ray astronomy; it is regularly observed by all gamma-ray telescopes 12

The spectrum and morphology of the PWN depend on the structure of the post-shock magnetohydrodynamic (MHD) flow. Observations in the X-ray energy band with the Chandra X-ray Observatory (Chan$d r a)^{13}$ revealed a complex structure consisting of a bright torus and a narrow jet emerging in the direction perpendicular to the torus plane (see Fig. 1, right). The X-ray structure suggests that the underlying MHD flow is nearly axisymmetric. The apparent deviation from this symmetry in the X-ray image is mostly due to Doppler boosting, which enhances the X-ray emission of fluid elements moving towards us. MHD instabilities developing in the post-shock region also alter the symmetry, but at a less important level.

In the model we use here (see ref ${ }^{14}$ and the Supplementary Information), which is based on the seminal work of Kennel and Coronit:15[16, the key observational features are reproduced by approximating the pulsar wind by a two-dimensional axisymmetric MHD flow propagating into a limited solid angle close to the torus plane. We combine this with a three-dimensional treatment of the radiation throughout the nebula to account for the Doppler boosting and orientation of the magnetic field. To this end, a high-energy particle distribution following a power law in energy forms at the termination shock. These particles, confined in fluid elements, are advected through the nebula by the MHD flow. Accounting for the evolution of the magnetic field, various target photon fields, and the changing rate of adiabatic cooling, the energy distribution of highenergy particles in each point of the nebula is computed. Taking into account local magnetic and photon fields as well as the Doppler boosting, this allows us to derive the surface brightness, which can then be directly compared with observations in different energy bands. We note that in the model the IC emission is dominated by upscattering of photons of the cosmic microwave background and the entire spectrum of synchrotron photons emitted by the same population of high-energy electrons (with two electron populations, the wind and the relic radio electrons, as detailled in ref.!17).

In our model, the structure of the nebula depends on three parameters: the radius of the termination shock, the magnetisation (defined as the ratio of electromagnetic to particle energy flux) of the wind at the termination shock, and the wind opening angle defined as the solid angle into which the bulk of the energy is ejected close to the torus plane. We note that there are other physical parameters of the system, like the spin-down luminosity of the pulsar, whose measured values are used as input to our model. With a 
suitable choice of parameters, the model reproduces the broadband spectral energy distribution (SED) well, as shown in Fig. 2. The model also predicts that the size of the nebula varies strongly with the energy of the emitting electrons. Since higher energy electrons suffer more severe radiation losses from both synchrotron radiation and IC scattering processes, they propagate shorter distances before they lose energy via radiation. This energy dependence is seen by current X-ray telescopes in the synchrotron radiation of the PWN up to $40 \mathrm{keV} \sqrt{18}$, and is also clearly seen in the data shown in Fig. 2, bottom. In the gamma-ray IC radiation domain, the PWN emission up to now appeared point-like and only upper limits on the size could be derived ${ }^{4 / 6}$ or evidence be claimed at $\mathrm{GeV}$ energies 19 .

With the new High Energy Stereoscopic System (H.E.S.S.) measurement shown in Fig. 1, we establish the IC PWN extension, at photon energies eight orders of magnitude above the previously highest energy morphology measurement of the Crab PWN in X-rays. This measurement of the IC extension of the Crab PWN provides a stringent test of our understanding of high-energy particle propagation and radiation models. Using 22 hours of observations collected over 6 years, we employ advanced analysis techniques to reconstruct the gamma-ray image of the Crab PWN. We compare this image to that of a simulated gamma-ray source, taking for each observation the exact hardware status of the H.E.S.S. telescopes and all observation conditions into account in the simulations. Simulating the exact state of the telescope system at any given time was not previously done, but allowed us here to increase the precision to the level needed to discover the extension of the Crab PWN at TeV gamma-ray energies (further details are given in Methods).

The comparison of data to simulations reveals that the nebula is extended (see Fig. 3). We can reproduce the data only by simulating an extended source. We do this by convolving the angular resolution function with a two-dimensional Gaussian with a best-fit value of $\sigma_{2 \mathrm{D}, \mathrm{G}}=52.2^{\prime \prime} \pm 2.9_{\mathrm{stat}}^{\prime \prime} \pm 6.6_{\mathrm{sys}}^{\prime \prime}$. The systematic uncertainty is related to the calibration and analysis method, to the spectral shape used to simulate the angular resolution, and to the fit method. The resulting radial distribution of gamma rays compared to the simulated angular resolution function, as well as the resolution function convolved with a Gaussian, is shown in Fig. 3. The event distribution is clearly incompatible with a point-like source, while a convolution of the resolution function with a Gaussian drastically improves the fit to the data by 9 standard deviations. We therefore conclude that we measure the Crab nebula as a substantially extended gamma-ray source at photon energies above $700 \mathrm{GeV}$.

The extension we measure is smaller than that seen in ultraviolet (UV) light and significantly larger than that seen in hard X-rays (see Fig. 1). This can be understood by considering the energies of the electrons producing the synchrotron and IC emission, respectively. As shown in Fig. 2, lower energy electrons are emitting the UV synchrotron photons, medium energy electrons are emitting the IC gamma rays, and higher energy electrons are responsible for the synchrotron X-ray emission measured in the Chandra image. Thus, the difference in size in the different wavebands is compatible with the energy dependent radiation losses of the parent electrons discussed above: higher energy electrons propagate shorter distances than lower energy ones (see Fig. 2 bottom).

By splitting our data into two parts, below and above $5 \mathrm{TeV}$, we have also searched for energy dependent changes of the $\mathrm{TeV}$ gamma-ray extension. Such changes are ultimately also expected to show up in $\mathrm{TeV}$ gamma-ray data. While the Crab PWN is significantly extended in both energy bins, our data are currently not precise enough to establish this energy dependence of the PWN extension.

As Fig. 2 (top) shows, the PWN morphology reflecting an electron energy range of $1-10 \mathrm{TeV}$ is now probed for the first time with the new H.E.S.S. image. The majority of the photons measured (dark purple vertical band) probes this electron energy range, which is inaccessible via $100 \mathrm{eV}$ synchrotron emission due to absorption by interstellar matter. The measurement of the TeV gamma-ray morphology of the Crab PWN is therefore the only way to trace such $1-10 \mathrm{TeV}$ electrons.

While the simplest one-dimensional MHD simulations 14 are known to reproduce the basic characteris- 
tics of the Crab PWN, we have now verified that with the model we use ${ }^{14}$ we can consistently reproduce the measured synchrotron and IC morphology of the PWN including our new measurement (see Fig. 2 , bottom). We find a best-fit model for the wind solid angle of $\sim 6.5$ steradians and a termination shock radius of $\sim 0.13$ parsec, both in good agreement with the Chandra X-ray datd $13 \mid 20$. The magnetisation of the wind is found to be $\sim 0.5 \%$, and is thus at the same level as previously found to reproduce the SED and synchrotron morphology ${ }^{17} \mid 21$. We note that the magnetisation value is affected by the assumed radial flow velocity and a lack of turbulence in our approach and can therefore not be directly compared to higher dimensional MHD simulations 22 (see also the Supplementary Information).

With this measurement we establish the extension of the Crab nebula at $\mathrm{TeV}$ gamma-ray energies and provide a new probe of the distribution of $1-10 \mathrm{TeV}$ electrons. This closes a gap in the multi-wavelength

coverage of this icon of high-energy astrophysics and provides a non-trivial test of our understanding of particle propagation and photon emission at very high energies.

\section{References}

1. Hester, J. J. The Crab Nebula: An Astrophysical Chimera. Annu. Rev. Astron. Astrophys. 46, 127-155 (2008).

2. Bühler, R. \& Blandford, R. The surprising Crab pulsar and its nebula: a review. Rep. Progr. Phys. 77, 066901 (2014).

3. Weekes, T. C. et al. Observation of $\mathrm{TeV}$ gamma rays from the Crab nebula using the atmospheric Cerenkov imaging technique. Astrophys. J. 342, 379-395 (1989).

4. Aharonian, F. A. et al. Optimizing the angular resolution of the HEGRA telescope system to study the emission region of VHE gamma rays in the Crab Nebula. Astron. Astrophys. 361, 1073-1078 (2000).

5. Aharonian, F. A. et al. Observations of the Crab nebula with HESS. Astron. Astrophys. 457, 899-915 (2006).

6. Albert, J. et al. VHE $\gamma$-Ray Observation of the Crab Nebula and its Pulsar with the MAGIC Telescope. Astrophys. J. 674, 1037-1055 (2008).

7. Meagher, K. Six years of VERITAS observations of the Crab Nebula. ICRC2015, Proc. Sci. 236, 792 (2016).

8. Abeysekara, A. U. et al. Observation of the Crab Nebula with the HAWC Gamma-Ray Observatory. Astrophys. J. 843, 39 (2017).

9. Abdo, A. A. et al. Fermi Large Area Telescope Observations of the Crab Pulsar And Nebula. Astrophys. J. 708, 1254-1267 (2010).

10. H. E. S. S. Collaboration et al. H.E.S.S. observations of the Crab during its March $2013 \mathrm{GeV}$ gamma-ray flare. Astron. Astrophys. 562, L4 (2014).

11. Aleksić, J. et al. Measurement of the Crab Nebula spectrum over three decades in energy with the MAGIC telescopes. J. High Energy Astrophys. 5, 30-38 (2015).

12. Archambault, S. et al. Gamma-ray observations under bright moonlight with VERITAS. Astropart. Phys. 91, 34-43 (2017).

13. Weisskopf, M. C. et al. Discovery of Spatial and Spectral Structure in the X-Ray Emission from the Crab Nebula. Astrophys. J. 536, L81-L84 (2000). 
14. Bogovalov, S. V. \& Khangoulian, D. V. On the origin of the torus and jet-like structures in the centre of the Crab Nebula. Mon. Not. R. Astron. Soc. 336, L53-L55 (2002).

15. Kennel, C. F. \& Coroniti, F. V. Confinement of the Crab pulsar's wind by its supernova remnant. Astrophys. J. 283, 694-709 (1984).

16. Kennel, C. F. \& Coroniti, F. V. Magnetohydrodynamic model of Crab nebula radiation. Astrophys. J. 283, 710-730 (1984).

17. Atoyan, A. M. \& Aharonian, F. A. On the mechanisms of gamma radiation in the Crab Nebula. Month. Not. R. Astron. Soc. 278, 525-541 (1996).

18. Madsen, K. K. et al. Broadband X-ray Imaging and Spectroscopy of the Crab Nebula and Pulsar with NuSTAR. Astrophys. J. 801, 66 (2015).

19. Ackermann, M. et al. The Search for Spatial Extension in High-latitude Sources Detected by the Fermi Large Area Telescope. Astrophys. J. 237, 32 (2018).

20. Weisskopf, M. C., Elsner, R. F., Kolodziejczak, J. J., O'Dell, S. L. \& Tennant, A. F. Unraveling the Geometry of the Crab Nebula's "Inner Ring". Astrophys. J. 746, 41 (2012).

21. Meyer, M., Horns, D. \& Zechlin, H.-S. The Crab Nebula as a standard candle in very high-energy astrophysics. Astron. Astrophys. 523, A2 (2010).

22. Del Zanna, L., Amato, E. \& Bucciantini, N. Axially symmetric relativistic MHD simulations of Pulsar Wind Nebulae in Supernova Remnants. On the origin of torus and jet-like features. Astron. Astrophys. 421, 1063-1073 (2004).

23. Komissarov, S. S. \& Lyubarsky, Y. E. Synchrotron nebulae created by anisotropic magnetized pulsar winds. Mon. Not. R. Astron. Soc. 349, 779-792 (2004).

24. Bucciantini, N., Thompson, T. A., Arons, J., Quataert, E. \& Del Zanna, L. Relativistic magnetohydrodynamics winds from rotating neutron stars. Mon. Not. R. Astron. Soc. 368, 1717-1734 (2006).

25. Del Zanna, L., Volpi, D., Amato, E. \& Bucciantini, N. Simulated synchrotron emission from pulsar wind nebulae. Astron. Astrophys. 453, 621-633 (2006).

26. Volpi, D., Del Zanna, L., Amato, E. \& Bucciantini, N. Non-thermal emission from relativistic MHD simulations of pulsar wind nebulae: from synchrotron to inverse Compton. Astron. Astrophys. 485, 337-349 (2008).

27. Porth, O., Komissarov, S. S. \& Keppens, R. Solution to the sigma problem of pulsar wind nebulae. Mon. Not. R. Astron. Soc. 431, L48-L52 (2013).

28. Dubner, G. et al. Morphological Properties of the Crab Nebula: A Detailed Multiwavelength Study Based on New VLA, HST, Chandra, and XMM-Newton Images. Astrophys. J. 840, 82 (2017).

29. Macías-Pérez, J. F., Mayet, F., Aumont, J. \& Désert, F.-X. Global Spectral Energy Distribution of the Crab Nebula in the Prospect of the Planck Satellite Polarization Calibration. Astrophys. J. 711, 417-423 (2010).

30. Mezger, P. G., Tuffs, R. J., Chini, R., Kreysa, E. \& Gemuend, H.-P. Maps of Cassiopeia A and the Crab Nebula at lambda 1.2 MM. Astron. Astrophys. 167, 145-150 (1986).

31. Bandiera, R., Neri, R. \& Cesaroni, R. The Crab Nebula at $1.3 \mathrm{~mm}$. Evidence for a new synchrotron component. Astron. Astrophys. 386, 1044-1054 (2002).

32. Veron-Cetty, M. P. \& Woltjer, L. Spectrophotometry of the continuum in the Crab Nebula. Astron. Astrophys. 270, 370-378 (1993). 
33. Hennessy, G. S. et al. Ultraviolet Imaging Telescope observations of the Crab Nebula. Astrophys. J. 395, L13-L16 (1992).

34. Wu, C.-C. Interstellar extinction and ultraviolet flux distribution of the Crab Nebula. Astrophys. J. 245, 581-588 (1981).

35. Kirsch, M. G. et al. Crab: the standard x-ray candle with all (modern) x-ray satellites. Proc. SPIE 5898, 22-33 (Aug. 2005).

36. Jourdain, E. \& Roques, J. P. The High-Energy Emission of the Crab Nebula from $20 \mathrm{keV}$ TO $6 \mathrm{MeV}$ with Integral SPI. Astrophys. J. 704, 17-24 (2009).

37. Buehler, R. et al. Gamma-Ray Activity in the Crab Nebula: The Exceptional Flare of 2011 April. Astrophys. J. 749, 26 (2012).

38. Aharonian, F. et al. The Crab Nebula and Pulsar between $500 \mathrm{GeV}$ and $80 \mathrm{TeV}$ : Observations with the HEGRA Stereoscopic Air Cerenkov Telescopes. Astrophys. J. 614, 897-913 (2004).

Acknowledgements: The support of the Namibian authorities and of the University of Namibia in facilitating the construction and operation of H.E.S.S. is gratefully acknowledged, as is the support by the German Ministry for Education and Research (BMBF), the Max Planck Society, the German Research Foundation (DFG), the Helmholtz Association, the Alexander von Humboldt Foundation, the French Ministry of Higher Education, Research and Innovation, the Centre National de la Recherche Scientifique (CNRS/IN2P3 and CNRS/INSU), the Commissariat à l'énergie atomique et aux énergies alternatives (CEA), the U.K. Science and Technology Facilities Council (STFC), the Knut and Alice Wallenberg Foundation, the National Science Centre, Poland grant no. 2016/22/M/ST9/00382, the South African Department of Science and Technology and National Research Foundation, the University of Namibia, the National Commission on Research, Science \& Technology of Namibia (NCRST), the Austrian Federal Ministry of Education, Science and Research and the Austrian Science Fund (FWF), the Australian Research Council (ARC), the Japan Society for the Promotion of Science and by the University of Amsterdam. We appreciate the excellent work of the technical support staff in Berlin, Zeuthen, Heidelberg, Palaiseau, Paris, Saclay, Tbingen and in Namibia in the construction and operation of the equipment. This work benefited from services provided by the H.E.S.S. Virtual Organisation, supported by the national resource providers of the EGI Federation.

Author Contributions: D. Berge, J. Hahn, M. Holler, D. Khangulyan, D. Parsons have analysed and interpreted the data, and prepared the manuscript. The whole H.E.S.S. collaboration has contributed to the publication with involvement at various stages ranging from the design, construction and operation of the instrument, to the development and maintenance of all software for data handling, data reduction and data analysis. All authors have reviewed, discussed, and commented on the present results and on the manuscript.

Author Information: Author Information Reprints and permissions information is available at www. nature.com/reprints. The authors declare no competing financial interests. Correspondence and requests for materials should be addressed to the H.E.S.S. Collaboration (contact.hess@hess-experiment.eu). 

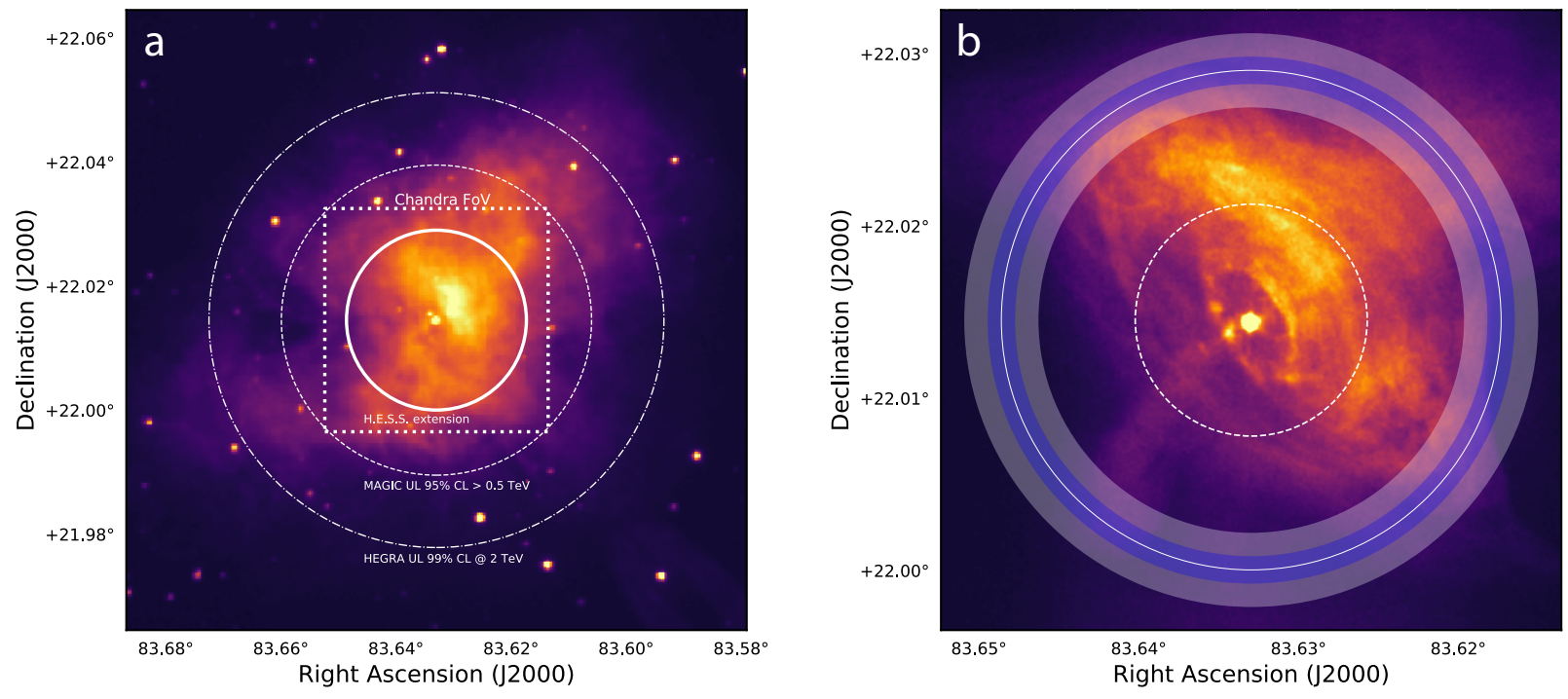

Figure 1: Images of the Crab nebula. a: UV $(\lambda=291 \mathrm{~nm})$ image recorded with the Optical-UV Monitor onboard XMM-Newton ${ }^{28}$. The MAGIC and HEGRA extension upper limits of $2.2^{\sqrt{6}}$ and $1.5^{\sqrt{4}}$ are drawn as dash-dotted and dashed lines, respectively. The extent of the sky region shown in $\mathbf{b}$ is indicated as dotted square, and the H.E.S.S. extension (two-dimensional Gaussian $\sigma$ corresponding to $39 \%$ of the measured events) is drawn as a solid circle. All circles are centred on the Crab pulsar position for illustration purposes, in the fit procedure determining the H.E.S.S. extension described in the main text the centroid position is left free. b: Chandra X-ray image ${ }^{13}$ (courtesy of M. C. Weisskopf and J. J. Kolodziejczak). The H.E.S.S. extension is shown as solid white circle overlaid on top of shaded annuli indicating the statistical and systematic uncertainties of our measurement. The Chandra extension, corresponding to $39 \%$ of the X-ray photons, is given as dashed white circle. 

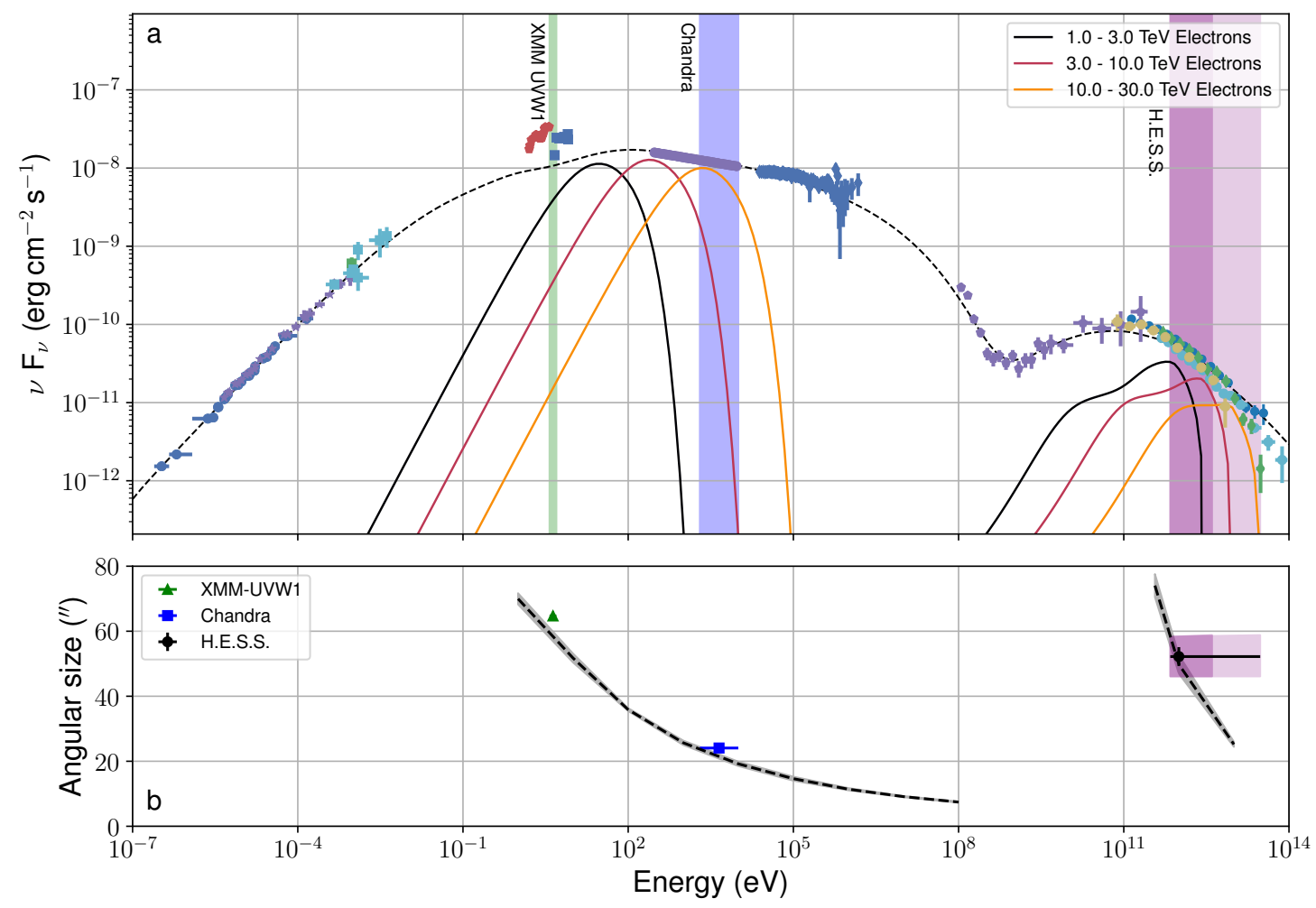

Figure 2: Spectral energy distribution (SED) along with the measured and predicted extensions of the Crab pulsar wind nebula. a: The SED is shown as dashed line. To illustrate the contribution of electrons of different energies to the radiation, the coloured lines show the synchrotron and IC radiation for electrons in the energy bands $1-3 \mathrm{TeV}$ (black), 3-10 TeV (red), and 10-30 TeV (yellow). The vertical bands indicate the measurement ranges of instruments in the UV (green), X-ray (blue), and TeV gamma-ray regime (purple). The dark purple part of the H.E.S.S. band indicates the energy range covered by $90 \%$ of the measured gamma-ray photons. The range of the remaining $10 \%$ of the highest energy photons is given as the light purple band. The data points from low to high energies are taken from refs $5 / 7 \sqrt[29]{38}$. Note that in the optical domain, the data points are above the SED indicative of a substantial contribution from thermal emission. b: The predicted (dashed line and grey shaded area, corresponding to the uncertainty) and measured (markers) extensions are plotted for various photon energies. The predicted extensions are the best-fit values of our model to the Chandra and H.E.S.S. data; the grey shaded uncertainty band results from up and down variations of 1 standard deviation of the fit parameters. The measured UV and X-ray extensions are determined by convolving the respective PWN images with the H.E.S.S. PSF and applying the same likelihood fit procedure described in the main text. The purple boxes indicate the H.E.S.S. energy range, their vertical size corresponds to the systematic uncertainty. All error bars are 1 standard deviation. 

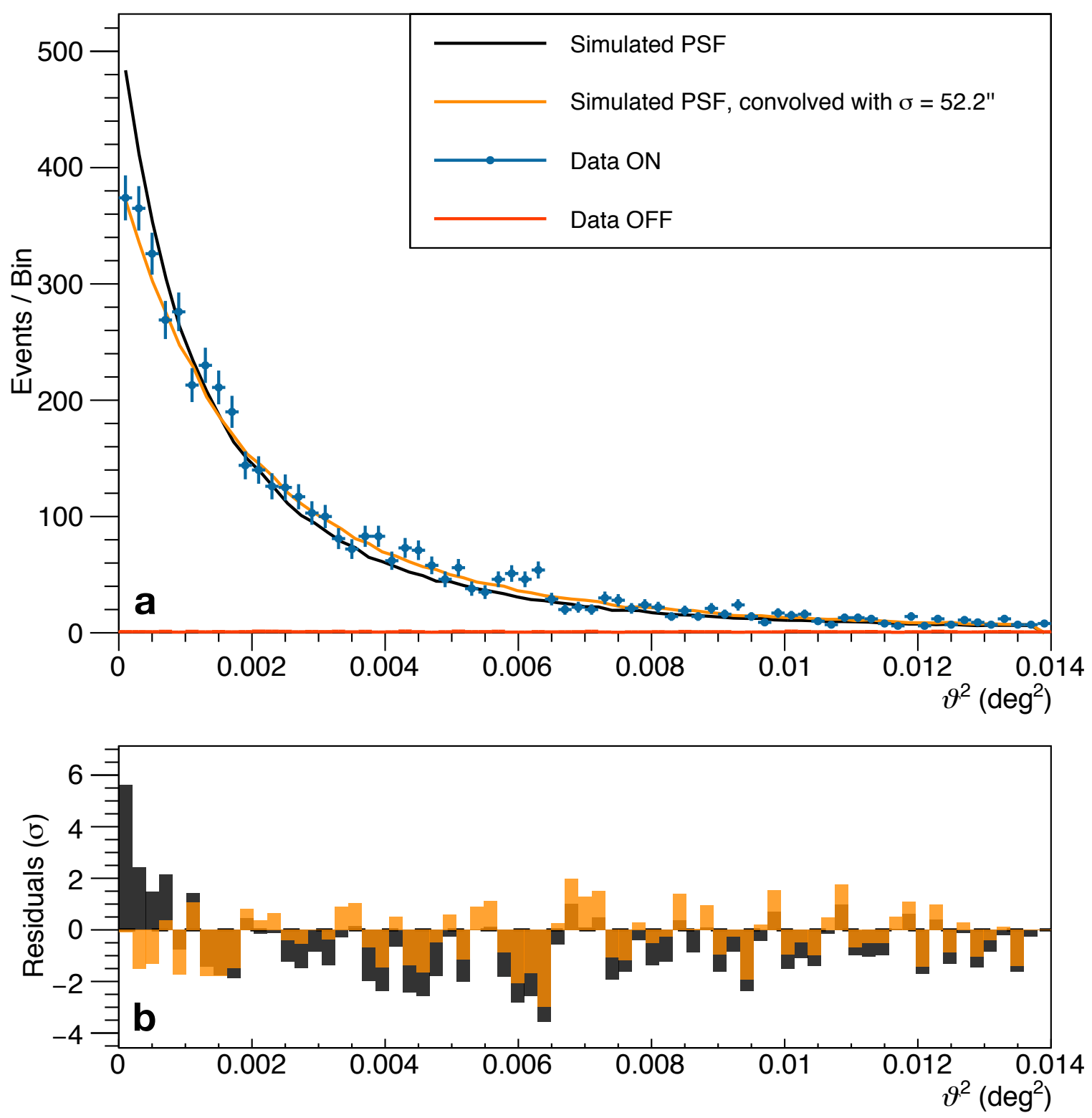

Figure 3: a: Histogram of reconstructed directions of gamma rays from the Crab nebula (Data ON, blue). The estimated background determined in empty regions of the sky is also shown (Data OFF, red). For comparison, the simulated angular resolution function (point spread function, PSF, black) for this dataset as well as the function convolved with the best-fit Gaussian (yellow) are shown. The error bars are 1 standard deviation.b: Significance of the bin-wise deviation $($ Data $-M C)$ of the data when compared to the PSF (black) and the convolved one (orange). 


\section{Methods}

The dataset used here was recorded with the High Energy Stereoscopic System (H.E.S.S.) array of telescopes. H.E.S.S. is an array of five imaging atmospheric Cherenkov telescopes. Such telescopes reconstruct cosmic gamma rays by recording images of Cherenkov light of the air showers that develop when a cosmic gamma ray smashes into the atmosphere. Such air showers are cascades of secondary charged particles, mostly electrons and positrons, which are created when gamma rays penetrate the atmosphere. The charged particles emit Cherenkov light, which in turn can be used to reconstruct the direction and energy of the primary gamma ray with telescopes like the H.E.S.S. array. The system consists of four telescopes with $108 \mathrm{~m}^{2}$ mirror area and $15 \mathrm{~m}$ focal length and a single $614 \mathrm{~m}^{2}$ telescope of $36 \mathrm{~m}$ focal length. H.E.S.S. is situated in the Khomas highlands of Namibia and is in the five-telescope configuration for observations near zenith sensitive to gamma-ray photons in the energy range from around $50 \mathrm{GeV}$ to around $50 \mathrm{TeV}$. The analysis presented here uses only data from the four small telescopes (which have a larger energy threshold of $100 \mathrm{GeV}$ near zenith). As the Crab nebula is such an important gamma-ray source it is regularly monitored by H.E.S.S. From this large monitoring dataset recorded over the course of 10 years, 22 hours of observations fulfil tight quality selection criteria aimed at optimising the angular resolution of the system and are used in this study (see Supplementary Table 1).

The data were analysed with the analysis technique introduced in ref ${ }^{1}$. This method is based on a semianalytical air-shower model, which is fit to the recorded air-shower images to yield the primary gamma-ray direction and energy. To improve the angular resolution of the standard analysis configuration, only well reconstructed gamma-ray candidates are considered further.

The analysis was conducted in three ranges in reconstructed energy, once using all events reconstructed between 0.7 and $30 \mathrm{TeV}$, and once in two separate energy bins from 0.7 to $5 \mathrm{TeV}$ and from 5 to $30 \mathrm{TeV}$. These three ranges are listed together with their respective detection significances of the Crab nebula as calculated with Eq. 17 of ref. ${ }^{2}$ and the respective angular resolutions in Supplementary Table 2.

For the subsequent morphology fit, two maps are produced: One containing all gamma-ray candidates (ON map), and one with the gamma-ray-like background, estimated with an improved version of the ring background technique ${ }^{3}$, which automatically adapts the ring size. The bin size of the maps is $0.01^{\circ} \times 0.01^{\circ}$, well below the width of the point spread function (PSF). We have verified that smaller bin sizes have no influence on the subsequent results. For visualisation purposes, the projected distribution of resulting events as a function of squared angular distance $\left(\vartheta^{2}\right)$ to the centroid of the measured gamma-ray excess is also calculated. This distribution for the $0.7<E<30 \mathrm{TeV}$ energy range is shown in Fig. 3 . The best-fit position in J2000 coordinates is $\alpha=5 \mathrm{~h} 34 \mathrm{~m} 30.9 \mathrm{~s} \pm(0.1 \mathrm{~s})_{\text {stat }} \pm(1.3 \mathrm{~s})_{\text {sys }}, \delta=+22^{\circ} 00^{\prime} 44.5^{\prime \prime} \pm 1.1_{\text {stat }}^{\prime \prime} \pm 20_{\text {sys }}^{\prime \prime}$ (systematic error from ref. ${ }^{\sqrt[4]{3}}$ ), which is within uncertainties compatible with the Crab pulsar location.

With dedicated Monte-Carlo (MC) simulations of the data-set, including the actual instrument and observation conditions at the time of the observations and using a power-law energy distribution ${ }^{5}$, we reweight the simulated events to mimic the shape of the Crab nebula's energy spectrum and analyse them with the same algorithms and analysis configurations as the actual data. The resulting $\vartheta^{2}$ histogram of this MC analysis serves as the PSF for this source and data-set and is also shown in the upper panel of Fig. 3. The $68 \%$ and $90 \%$ containment radii of our PSF are given in Supplementary Table 2.

As apparent in Fig. 3, the PSF is highly inconsistent with the distribution of the gamma-ray excess counts. The residuals in the lower panel indicate clearly that the data are shallower than the PSF. To study this further, we perform a two-dimensional morphology fit with Sherp $a^{6}$, using the ON map, the background 
map, and the simulated PSF. The PSF is convolved with a two-dimensional radially symmetric Gaussian:

$$
\frac{\mathrm{d} P}{\mathrm{~d} \vartheta^{2}}=\frac{1}{2 \sigma_{2 \mathrm{D}, \mathrm{G}}^{2}} \cdot \exp \left(-\frac{\vartheta^{2}}{2 \sigma_{2 \mathrm{D}, \mathrm{G}}^{2}}\right) .
$$

To quantify the compatibility of the data and the convolved PSF, a likelihood value is calculated and minimised. The best-fit extension is found to be $\sigma_{2 \mathrm{D}, \mathrm{G}}=52.2^{\prime \prime} \pm 2.9_{\mathrm{stat}}^{\prime \prime} \pm 6.6_{\mathrm{sys}}^{\prime \prime}$, with a preference of an extension of the Crab nebula over a point-source assumption of TS $\approx 83$. As systematic uncertainty of the extension we quote the quadratic sum of uncertainties related to the calibration and analysis method, to the spectral shape used to re-weight the MC PSF, and to the fit method.

The resulting best-fit convolution is also plotted in Fig. 3 It clearly provides a good description of the data both in the upper panel and the residuals in the lower panel.

To verify the robustness of our result, we applied the analysis using time-dependent simulations to two other bright and highly significant extragalactic point-like gamma-ray sources, the active galactic nuclei PKS 2155-304 and Markarian 421. As illustrated in Supplementary Figure 1, both sources appear to be point-like, while the Crab PWN data is very clearly extended. Upper limits on the extension of PKS 2155-304 and Markarian 421 are shown in Supplementary Figure 2. These are well below the measured extension of the Crab nebula. We emphasise that Markarian 421 culminates at large zenith angles of $\phi>60^{\circ}$ at the H.E.S.S. site (as opposed to $\phi \approx 47^{\circ}$ for the Crab nebula culmination), making this source a particularly convincing test of our PSF understanding, since larger zenith angle observations have larger systematic PSF uncertainties. As we also show in Supplementary Figure 2, we tested the Crab nebula data-set for a zenith-angle dependence by splitting the observations in two data-sets above and below $46^{\circ}$. The measured extensions are compatible with each other.

The results have also been cross-checked with an independent calibration, reconstruction, and analysis method ${ }^{7}$. We find this second extension measurement slightly larger than our nominal value (see Supplementary Figure 2), and use this difference as an estimate of the systematic uncertainty related to the analysis method.

Data and Code Availability Statement: The raw data and the code used in this study are not public but belong to the H.E.S.S. collaboration. All derived higher level data that are shown in plots will be made available on the H.E.S.S. collaboration's web site upon publication of this study.

\section{Additional references}

1. De Naurois, M. \& Rolland, L. A high performance likelihood reconstruction of $\gamma$-rays for imaging atmospheric Cherenkov telescopes. Astropart. Phys. 32, $231-252$ (2009).

2. Li, T.-P. \& Ma, Y.-Q. Analysis methods for results in gamma-ray astronomy. Astrophys. J. 272, 317324 (1983).

3. Berge, D., Funk, S. \& Hinton, J. Background modelling in very-high-energy $\gamma$-ray astronomy. Astron. Astrophys. 466, 1219-1229 (2007).

4. Gillesen, S. Sub-Bogenminuten-genaue Positionen von TeV-Quellen mit H.E.S.S. German. PhD Dissertation http: //www. bsz-bw. de/cgi-bin/xums. cgi? SWB11244050 (2004).

5. Holler, M., Chevalier, J., Lenain, J.-P., de Naurois, M. \& Sanchez, D. Run-Wise Simulations for Imaging Atmospheric Cherenkov Telescope Arrays. ICRC2017, Proc. Sci. 301, 755 (2018).

6. Freeman, P., Doe, S. \& Siemiginowska, A. Sherpa: a mission-independent data analysis application. Proc. SPIE 4477, 76-87 (2001). 
7. Parsons, R. D. \& Hinton, J. A. A Monte Carlo template based analysis for air-Cherenkov arrays. Astropart. Phys. 56, 26-34 (2014). 


\section{Supplementary Information}

\section{Analysis and Results}

Figures 4 and 5 demonstrate the robustness of our Crab nebula extension measurement. In Fig. 4 we show that we can clearly separate extragalactic point-like gamma-ray sources like the active galactic nuclei PKS 2155-304 and Markarian 421 from the extended Crab pulsar wind nebula. In Fig. 5 we show in addition that the measured extension is robust under variations of observation conditions and analysis chains.

Tables 1 and 2 detail the dataset and gamma-ray energy regimes investigated in this study. 


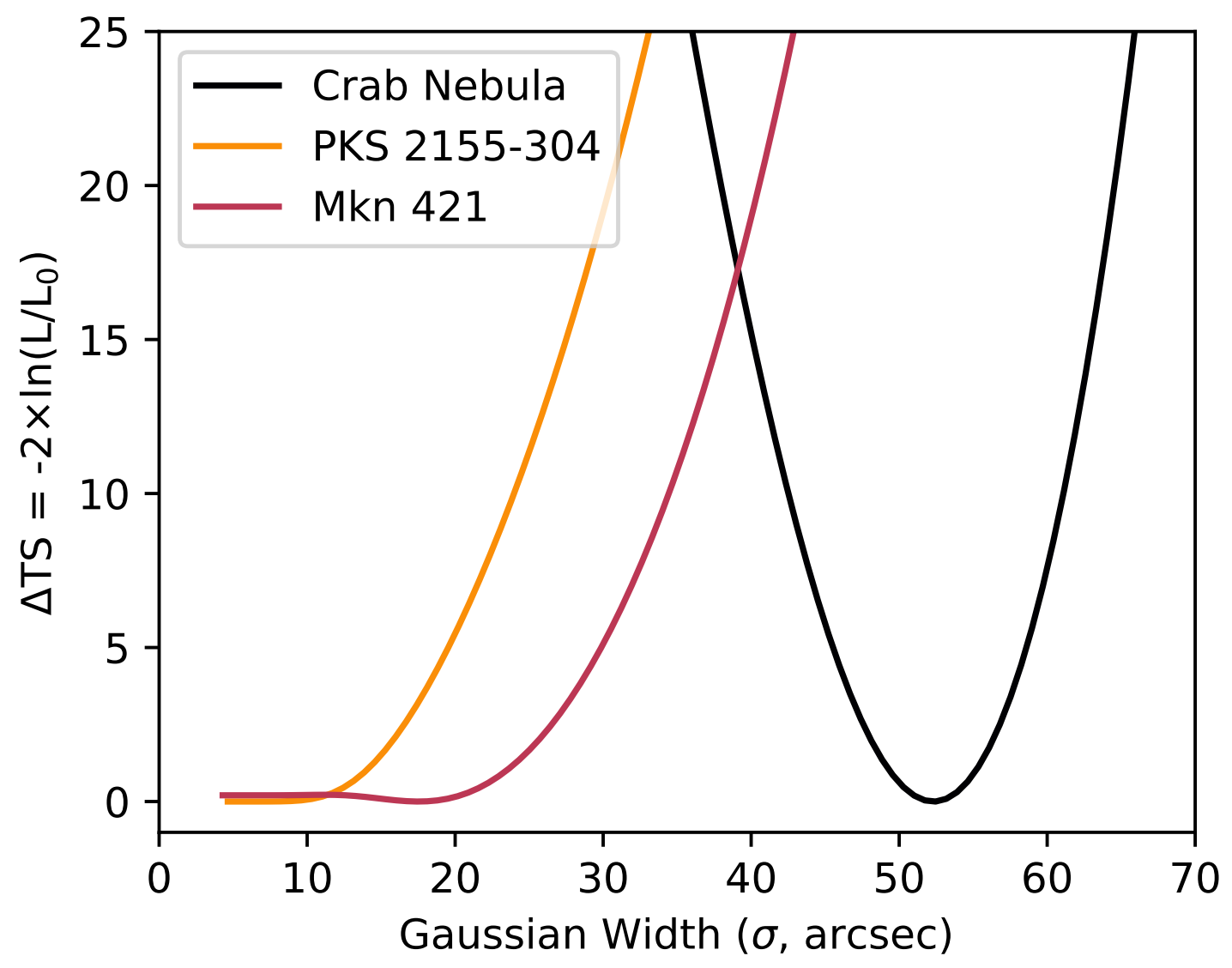

Figure 4: Results of the extension fits. Shown are the extension profiles of the difference in test statistics $\left(\Delta T S=-2 \times \ln \left(L / L_{0}\right)\right)$ for the three sources evaluated here. The likelihood $L_{0}$ corresponds to the value at the respective minimum. 


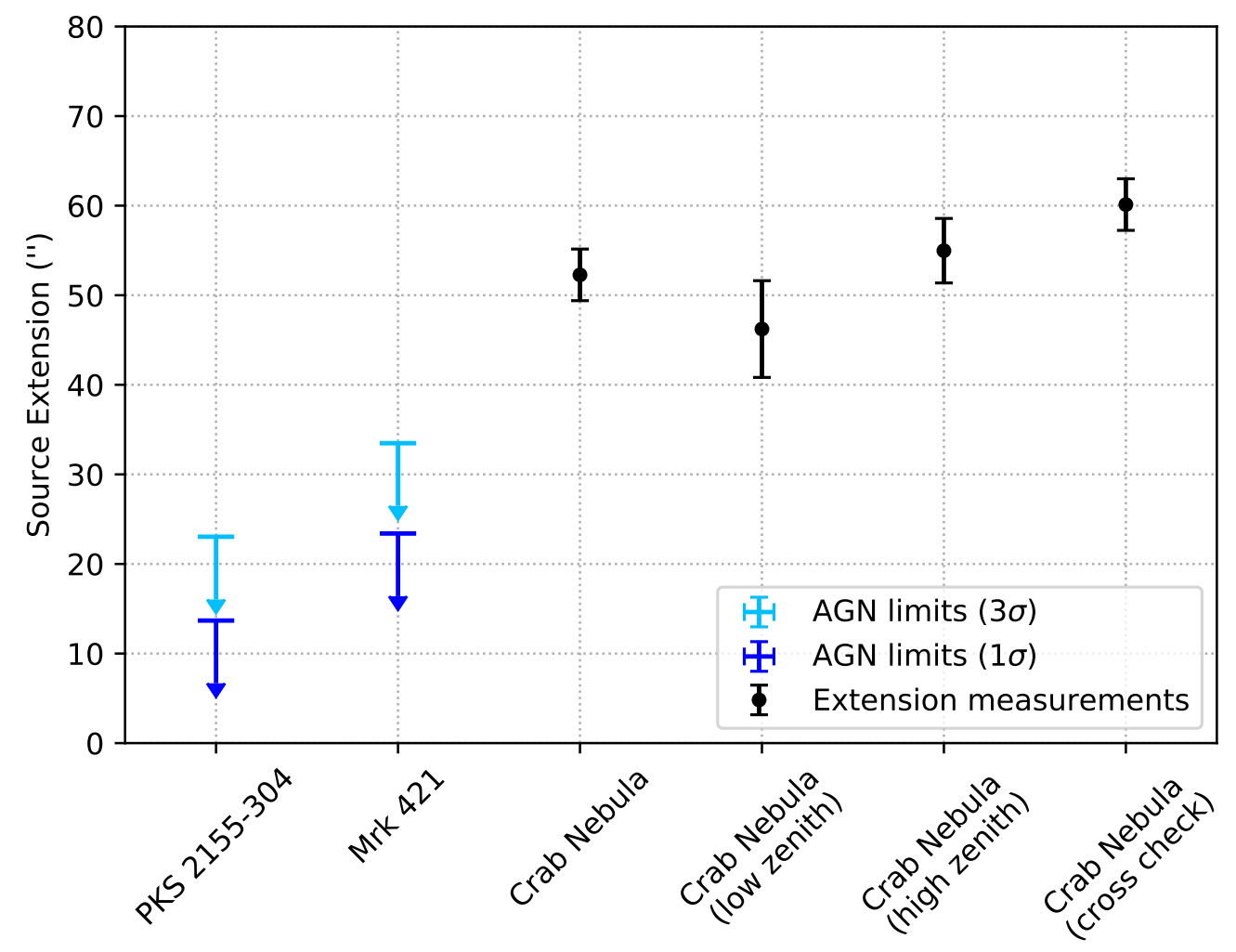

Figure 5: Systematic checks of the extension measurement. We present the derived extension upper limits of PKS 2155-304 and Markarian 421. The blue and cyan symbols are the 1 and 3 standard deviations upper limits, respectively. Shown in addition are the measured extension of the Crab nebula and systematic checks. The low and high zenith angle band correspond to $44-46^{\circ}$ and $46-55^{\circ}$, respectively. The error bars are statistical uncertainties (1 standard deviation). 


\begin{tabular}{lccc}
\hline year & $\begin{array}{c}\text { mean offset } \\
\text { (degrees) }\end{array}$ & $\begin{array}{c}\text { mean zenith angle } \\
\text { (degrees) }\end{array}$ & $\begin{array}{c}\text { livetime } \\
\text { (hours) }\end{array}$ \\
\hline 2004 & 0.5 & 47 & 11.3 \\
2007 & 0.5 & 46 & 3.6 \\
2008 & 0.5 & 48 & 1.3 \\
2009 & 0.5 & 46 & 7.1 \\
all & 0.5 & 47 & 22.3 \\
\hline
\end{tabular}

Table 1: Overview of the H.E.S.S. observation campaigns used in this study. The livetime given in hours corresponds to the data fulfilling data quality requirements.

\begin{tabular}{lccc}
\hline Energy Range & Detection Significance & $R_{68}\left(^{\circ}\right)$ & $R_{90}\left({ }^{\circ}\right)$ \\
\hline $0.7 \mathrm{TeV}<E<30 \mathrm{TeV}$ & $136.8 \sigma$ & 0.052 & 0.088 \\
$0.7 \mathrm{TeV}<E<5 \mathrm{TeV}$ & $131.4 \sigma$ & 0.053 & 0.088 \\
$5 \mathrm{TeV}<E<30 \mathrm{TeV}$ & $39.2 \sigma$ & 0.043 & 0.081 \\
\hline
\end{tabular}

Table 2: Definition of the energy bands used for the analysis. The detection significances of the Crab nebula and the angular resolutions expressed by the $68 \%$ and $90 \%$ containment radii $\left(R_{68}\right.$ and $\left.R_{90}\right)$ of the simulated PSF are also given. 


\section{On the PWN Radiation Modelling}

\section{Introduction}

Modern models for PWN have been developed over the last forty years. They are based on the concept of a pulsar wind, an ultrarelativistic outflow that connects the pulsar magnetosphere to an outer nebula, which can be up to a few parsec in $\operatorname{size}^{1}$, and on an MHD treatment of the PWN (described in ref. ${ }^{2}$, called KC2 in the following). In this model, the transport and radiative cooling of high-energy particles is consistently described with the analytical solution for the underlying MHD flow. The KC2 model for the non-thermal particles in the nebula allowed computing the volume emissivity of synchrotron radiation. The emissivity appeared to be quite sensitive to the properties of the MHD flow, in particular to its magnetisation. The spectra predicted by the model agreed well with observations provided that the pulsar wind is weakly magnetised and ultra-relativistic. Later, ref ${ }^{[3}$ extended the approach of $\mathrm{KC} 2$ and computed self-consistently the IC emission of the high-energy particles. The spectra obtained agreed with observations in a vast range, from optical wavelengths to the very high energy gamma-ray band. This success gave strong support to MHD models and made a strong case for efficient acceleration of charged particles to very high energies by relativistic shock in PWNe.

The model of $\mathrm{KC} 2$ contains, however, an obvious shortcoming. It utilizes an internally inconsistent model since one-dimensional MHD models cannot include a toroidal magnetic field. This contradiction can be resolved with two- or three-dimensional MHD models. Moreover, there is another argument for a multidimensional MHD description. The energy flux in the pulsar wind should be highly anisotropic with the most significant fraction of energy released into a relatively small range of solid angles close to the equatorial plane 4 . Ref. ${ }^{4}$ suggested that a simple MHD model that utilizes the analytical solution of $\mathrm{KC} 2$, limited to a region close to the equatorial plane, can qualitatively reproduce the bright torus seen in the X-ray energy band with Chandra ${ }^{6}$. The formation of the jet-like plumes seen in these Chandra data of the Crab PWN is then likely caused by magnetic collimation ${ }^{5 / 7}$.

Further on, the model of KC2 was extended by numerical MHD calculations in $\mathrm{tw}^{8-10}$ and thre ${ }^{11}$ dimensions. Although the quantitative comparison of the three-dimensional numerical model with observational data has not yet been performed, many features of the numerical solution seem to have a clear association with some observed phenomena. In particular, X-ray wisps are robustly associated with MHD waves propagating in the nebula.

To verify the potential of the $\mathrm{TeV}$ gamma-ray data, which reveal the extension of the nebula, to constrain the allowed model parameter space, we performed numerical simulations of energy spectra and the morphology of the non-thermal electromagnetic emission. Such a study of a multi-dimensional parameter space demands a computationally efficient model. We have therefore adopted a one-dimensional MHD model as developed in KC2. Since it is well known from the Chandra X-ray data of the central part of the Crab nebula that the anisotropy of the pulsar wind strongly influences the distribution of high-energy particles, we introduced an additional parameter, $\Delta \Omega$, the solid angle into which the wind outflow propagates. Thus the wind propagation region occupies a disk-like volume around the equatorial plane. The region outside the disk is occupied by plasma that does not yield any vital contribution to the X-ray emission and therefore we ignore it.

We note that the one-dimensional numerical approach we take should be considered as a phenomenological model in contrast to actual hypotheses represented by more realistic three-dimensional MHD simulations ${ }^{12}$. Consequently, parameters like the flow magnetisation parameter should be treated as internal parameters of the phenomenological model that cannot be directly compared to their values in two- or three-dimensional models. The flow magnetisation for example, due to the rigid flow geometry, tends to have smaller values in one-dimensional models, which are formally inconsistent with the values revealed with more detailed three-dimensional simulations. Applying a one-dimensional model is then still worthwhile as it demonstrates 
the potential of the $\mathrm{TeV}$ gamma-ray morphology data to constrain the model parameter and thus verify the consistency of the tested model. The phenomenological model used accurately accounts for processes governing the particle emission. It allows us to link different radiation domains (synchrotron, IC emission) and the energy-dependence of the emission volumes visible in these domains. Thus, a hypothetical inconsistency of the used phenomenological model with the $\mathrm{X}$ - and gamma-ray data should be considered as a serious challenge for all MHD models for the Crab Nebula. Finally, we note that when detailed three-dimensional simulations of the synthetic emissivity in the nebula will be available, an almost identical approach will help us to constrain the allowed parameter space for these models.

\section{MHD treatment}

In the framework of our model, the MHD flow in the disk depends on three parameters. The first important parameter is the wind magnetisation. This parameter determines the fraction of the pulsar spin-down losses carried away in the form of a Poynting flux

$$
L_{\mathrm{SD}}=\Delta \Omega n_{1} \gamma_{1} u_{1} r_{\mathrm{TS}}^{2} m c^{3}(1+\sigma) .
$$

Here $L_{\mathrm{SD}}$ is the pulsar spin-down (SD) losses, $m$ is the electron rest mass, and $c$ is the velocity of light. The parameters with subscript 1 describe the flow upstream of the termination shock (TS): $n_{1}, \gamma_{1}$, and $u_{1}$ are the plasma density, the bulk Lorentz factor, and the four-velocity, respectively. The wind magnetisation parameter is then:

$$
\sigma=\frac{B_{1}^{2}}{4 \pi n_{1} \gamma_{1} u_{1} r_{\mathrm{TS}}^{2} m c^{2}} .
$$

The TS radius, $r_{\mathrm{TS}}$, the wind opening angle, $\Delta \Omega$, and the wind magnetisation are the three parameters that determine the model MHD solution. Chandra X-ray observations constrain the $r_{\mathrm{TS}} \simeq 0.1-0.15 \mathrm{pc}$ at the pulsar wind equatorial plane. Depending on the flow zenith angle, $\theta$, the distance between the pulsar and

the TS can change considerably. However, since the bulk of the emission is produced close to the equatorial plane, we assume that the Chandra measurements define the physical range for the model parameter $r_{\mathrm{TS}}$.

The wind opening angle is related to an anisotropy of the energy flux in the pulsar wind, but other factors may also have a considerable impact on it. For example, in the framework of more realistic two- or three-dimensional MHD models, the shocked pulsar wind can be significantly deflected towards the equatorial plane. This effect cannot be consistently accounted for by the one-dimensional model used here. Instead, we allow the model parameter $\Delta \Omega$ to also take smaller values than anticipated by the expected energy anisotropy, which is expected to be proportional to $\sin ^{2} \theta$.

Downstream of the TS, approximating the magnetic field as toroidal, the flow dynamics is described by the following system of equations ${ }^{2}$, which describes conservation of particle flux, magnetic flux, adiabatic assumption, and total energy, respectively:

$$
\begin{aligned}
\frac{\mathrm{d}}{\mathrm{d} t}\left(\text { cnur }^{2}\right) & =0, \\
\frac{\mathrm{d}}{\mathrm{d} r}\left(\frac{r u B}{\gamma}\right) & =0, \\
\frac{\mathrm{d}}{\mathrm{d} r}\left(n u r^{2} e\right)+P \frac{\mathrm{d}}{\mathrm{d} r}\left(r^{2} u\right) & =0, \\
u \frac{\mathrm{d}}{\mathrm{d} r}(\gamma \epsilon)=\frac{\mathrm{d}}{\mathrm{d} r}\left[n u r^{2}\left(\gamma \mu+\frac{B^{2}}{4 \pi n \gamma}\right)\right] & =0 .
\end{aligned}
$$


Here $e$ is the specific internal energy per particle, $\mu$ is the specific enthalpy $(\mu=\epsilon+p)$, and $\epsilon$ is the sum of the specific electromagnetic and internal energy in the proper frame. $P$ is then the gas pressure and $p$ the specific pressure.

$\mathrm{KC} 2$ have shown that the combination of the four equations given above $(4)-(7)$ leads to the following expression:

$$
\left(1+u_{2}^{2} v^{2}\right)^{1 / 2}\left[\delta+\Delta\left(v z^{2}\right)^{-1 / 3}+\frac{1}{v}\right]=\gamma_{2}(1+\delta+\Delta)
$$

which determines the downstream flow velocity $u(z)=u_{2} v$ as a function of the dimensionless distance: $z=r / r_{\mathrm{TS}}$. The subscript 2 marks again the flow parameters downstream of the TS. The up- and downstream parameters are related through the Rankine-Hugoniot conditions. The dimensionless parameters $\delta$ and $\Delta$ are defined as

$$
\begin{aligned}
\delta & =\frac{4 \pi n_{2} \gamma_{2}^{2} m c^{2}}{B_{2}^{2}} \approx \frac{u_{2}}{u_{1} \sigma} \approx 0, \\
\Delta & \equiv \frac{16 \pi P_{2} \gamma_{2}^{2}}{B_{2}^{2}}=\left(\frac{1+\sigma}{\sigma}\right) \frac{u_{2}}{\gamma_{2}}-1 .
\end{aligned}
$$

Since the pulsar wind is expected to be ultra-relativistic, $\gamma_{1}>u_{1} \gg 1$, and since the downstream velocity is determined by the Rankine-Hugoniot conditions, the equation (8) depends effectively only on the magnetisation $\sigma$. The two other parameters that determine the normalisation factors are $r_{\mathrm{TS}}$ and $\Delta \Omega$, the characteristic length scale and the geometric extension of the emitting volume (that is, the flow).

\section{Non-thermal particles}

We assume that particles up to and beyond $\mathrm{TeV}$ energies in the Crab Nebula are accelerated at the pulsar wind TS. The acceleration process results in a fixed distribution of particles in the immediate vicinity of the TS. According to ref ${ }^{[}$, the spectral energy distribution of the Crab Nebula is well reproduced by a parent electron distribution following a broken power-law with exponential cutoff:

$$
n_{0}=\left.\frac{\mathrm{d} N}{\mathrm{~d} \varepsilon}\right|_{r=r_{\mathrm{TS}}}=\left\{\begin{array}{c}
A \varepsilon^{-p_{\mathrm{INJ}}} \exp \left(-\frac{\varepsilon}{\varepsilon_{\mathrm{CUT}}}\right), \varepsilon>\varepsilon_{\mathrm{b}} \\
A\left(\varepsilon / \varepsilon_{\mathrm{b}}\right)^{-3 / 2} \varepsilon_{\mathrm{b}}^{-p_{\mathrm{IN},},}, \varepsilon<\varepsilon_{\mathrm{b}}
\end{array}\right.
$$

where $\varepsilon$ is the electron energy, $A$ is a normalisation constant, and $\varepsilon_{\mathrm{CUt}}$ is the cutoff energy. Following ref. ${ }^{3}$, we adopted $\varepsilon_{\mathrm{CUT}}=2.5 \times 10^{15} \mathrm{eV}$ and $p_{\mathrm{INJ}}=2.4$. At the TS, the distribution normalisation, $A$, and the break energy, $\varepsilon_{\mathrm{b}}$, are adjusted so that the total number of particles and internal energy of the non-thermal distribution equal the values dictated by the Rankine-Hugoniot conditions.

The electron energy distribution in the flow changes with distance from the termination shock due to particle energy losses. We consider a differential volume element $\mathrm{d} V$ at distance $r$ from the TS:

$$
n(r, \varepsilon)=n\left(r=r_{\mathrm{TS}}, \varepsilon_{0}\right) \frac{\mathrm{d} \varepsilon_{0}}{\mathrm{~d} \varepsilon} \frac{\mathrm{d} V_{0}}{\mathrm{~d} V}=n_{0} \varphi \frac{\mathrm{d} \varepsilon_{0}}{\mathrm{~d} \varepsilon},
$$

where $n_{0}=n\left(r=r_{\mathrm{TS}}, \varepsilon_{0}\right)$ is the initial electron energy distribution, and $\varphi=\rho / \rho_{0}$ is the plasma compression (that is, a parameter determined with MHD simulations). The subscript 0 indicates the particles at TS $r=r_{\mathrm{TS}}$, which corresponds to the moment when the fluid element passes the TS and non-thermal particles are accelerated. 
The time evolution of particle energy $\varepsilon$ is described by the cooling equation:

$$
\frac{\mathrm{d} \varepsilon}{\mathrm{d} r}=\frac{1}{v} \dot{\varepsilon}(r, \varepsilon),
$$

where $\dot{\varepsilon}$ is the energy loss rate. In PWNe, synchrotron (SYN), inverse Compton (IC), and adiabatic (AD) energy losses represent the most important cooling channels:

$$
\dot{\varepsilon}(r, \varepsilon)=\dot{\varepsilon}_{\mathrm{SYN}}(r, \varepsilon)+\dot{\varepsilon}_{\mathrm{IC}}(r, \varepsilon)+\dot{\varepsilon}_{\mathrm{AD}}(r, \varepsilon) .
$$

Further computational simplification can be achieved if one adopts the Thompson approximation for IC cooling. In this case, one obtains

$$
\begin{aligned}
& \dot{\varepsilon}_{\mathrm{SYN}}+\dot{\varepsilon}_{\mathrm{IC}}=-a \varepsilon^{2}, \\
& a=\frac{4}{3} \frac{\sigma_{\mathrm{T}} c}{\left(m c^{2}\right)^{2}}\left(w_{\mathrm{PH}}+w_{\mathrm{B}}\right),
\end{aligned}
$$

where $\sigma_{\mathrm{T}}$ is the Thomson cross section, and $w_{\mathrm{PH}}$ and $w_{\mathrm{B}}$ are the energy densities of the target photons and the magnetic field, respectively. The adiabatic loss rate is given by

$$
\dot{\varepsilon}_{\mathrm{AD}}=\frac{v}{3} \frac{\mathrm{d} \ln \rho}{\mathrm{d} r} \varepsilon
$$

where $\rho$ is the plasma density in the fluid element. Equation (14) can now be rewritten by using equations (16) and $(17)$ as

$$
v \frac{\mathrm{d}}{\mathrm{d} r}\left(\frac{\rho^{1 / 3}}{\varepsilon}\right)=\rho^{1 / 3}(r) a(r) .
$$

Solving this equation, one obtains

$$
n(r, \varepsilon)=\varphi^{4 / 3}\left(\frac{\varepsilon_{0}}{\varepsilon}\right)^{2} n_{0},
$$

where $\varepsilon_{0}$ is the initial electron energy:

$$
\varepsilon_{0}=\varepsilon \frac{\varphi^{1 / 3}}{1-\varepsilon \lambda \rho^{-1 / 3}} .
$$

The parameter $\lambda$ accounts for radiative and adiabatic cooling and is defined as

$$
\lambda=\int_{r_{\mathrm{TS}}}^{r} \rho^{1 / 3}\left(r^{\prime}\right) a\left(r^{\prime}\right) \frac{\mathrm{d} r^{\prime}}{v\left(r^{\prime}\right)} .
$$

\section{Non-thermal radiation}

We aim to compute the spatial extension of the non-thermal emission in the nebula. Since the plasma emissivity varies considerably through the outflow, the emission specific intensity and the total specific emission should be computed by integrating over the line-of-sight (LoS) or the volume occupied by the outflow:

$$
I_{\nu}=\frac{\mathrm{d} E}{\mathrm{~d} t \mathrm{~d} \Omega \mathrm{d} \nu \mathrm{d} S}=\int_{\operatorname{LoS}} j_{\nu} \mathrm{d} \ell=\int_{\operatorname{LoS}}\left(\frac{\nu}{\nu^{\prime}}\right)^{2} j_{\nu^{\prime}}^{\prime} \mathrm{d} \ell,
$$


where the primed variables correspond to the co-moving frame of the plasma. In this case, the photon emission frequencies are affected by a Doppler boosting factor:

$$
\nu=\mathcal{D} \nu^{\prime}
$$

where $\mathcal{D}=1 /\left[\gamma\left(1-\boldsymbol{\beta} \hat{\boldsymbol{r}}_{\mathrm{OBS}}\right)\right], \boldsymbol{\beta}$ is the flow bulk velocity, and $\hat{\boldsymbol{r}}_{\mathrm{OBS}}$ is a unit vector pointing towards the observer. The dependence of the Lorentz factor on the flow direction implies that the computation of the emission should be performed in 3D geometry accounting for plasma bulk velocity in each point of the nebula.

In the case of synchrotron radiation it is more convenient to consider the emissivity in the fluid co-moving frame. In this frame one can use the energy distribution to compute the radiation:

$$
j_{\nu^{\prime}}^{\prime}=\int \frac{\mathrm{d} N_{\mathrm{SP}}^{\prime}}{\mathrm{d} t^{\prime} \mathrm{d} \nu^{\prime}} \frac{n^{\prime}\left(t^{\prime}, \boldsymbol{r}^{\prime}, \varepsilon^{\prime}\right)}{4 \pi} \mathrm{d} \varepsilon^{\prime} .
$$

Here, $\frac{\mathrm{d} N_{\mathrm{sp}}^{\prime}}{\mathrm{d} t^{\prime} \mathrm{d} \nu^{\prime}}$ represents the standard single-particle spectrum of synchrotron radiation.

In the case of IC radiation, it is more convenient to define the photon target in the laboratory frame, and consequently to compute the emission directly in this frame. One obtains the emission as

$$
j_{\nu}=\int \frac{\mathrm{d} N_{\mathrm{SP}}}{\mathrm{d} t \mathrm{~d} \nu} f\left(\boldsymbol{r}, p \hat{\boldsymbol{r}}_{\mathrm{OBS}}\right) p^{2} \mathrm{~d} p
$$

where $p=\sqrt{\varepsilon^{2}-m^{2}}$ is the particle momentum and $f$ is the Lorentz-invariant distribution function:

$$
\mathrm{d} N=f(\boldsymbol{r}, \boldsymbol{p}) \mathrm{d}^{3} \boldsymbol{r} \mathrm{d}^{3} \boldsymbol{p} .
$$

This function can be expressed through the energy distribution in the plasma co-moving frame as

$$
f\left(\boldsymbol{r}, p \hat{\boldsymbol{r}}_{\mathrm{OBS}}\right) \simeq \frac{c^{3} \mathcal{D}^{2}}{4 \pi \varepsilon^{2}} n^{\prime}(\boldsymbol{r}, \varepsilon / \mathcal{D}) .
$$

The single-particle spectrum of IC emission can be obtained through the angle averaged differential crosssection 13 as

$$
\frac{\mathrm{d} N_{\mathrm{SP}: \mathrm{IC}}}{\mathrm{d} t \mathrm{~d} \nu}=c \int n_{\mathrm{PH}}\left\langle\frac{\mathrm{d} \sigma_{\mathrm{IC}}}{\mathrm{d} \nu}\left(\varepsilon, \nu, \epsilon_{\mathrm{PH}}\right)\right\rangle \mathrm{d} \epsilon_{\mathrm{PH}},
$$

where the target photon density accounts for all important contributions like the cosmic microwave background, the far- and near-infrared, and a synchrotron-self-Compton contribution:

$$
n_{\mathrm{PH}}=n_{\mathrm{PH}, \mathrm{CMBR}}+n_{\mathrm{PH}, \mathrm{FIR}}+n_{\mathrm{PH}, \mathrm{NIR}}+n_{\mathrm{PH}, \mathrm{SSC}} .
$$

\section{Supplementary Information references}

1. Rees, M. J. \& Gunn, J. E. The origin of the magnetic field and relativistic particles in the Crab Nebula. Mon. Not. R. Astron. Soc. 167, 1-12 (1974).

2. Kennel, C. F. \& Coroniti, F. V. Magnetohydrodynamic model of Crab nebula radiation. Astrophys. J. 283, 710-730 (1984).

3. Atoyan, A. M. \& Aharonian, F. A. On the mechanisms of gamma radiation in the Crab Nebula. Month. Not. R. Astron. Soc. 278, 525-541 (1996). 
4. Bogovalov, S. V. \& Khangoulian, D. V. On the origin of the torus and jet-like structures in the centre of the Crab Nebula. Mon. Not. R. Astron. Soc. 336, L53-L55 (2002).

5. Lyubarsky, Y. E. On the structure of the inner Crab Nebula. Mon. Not. R. Astron. Soc. 329, L34-L36 (2002).

6. Weisskopf, M. C. et al. Discovery of Spatial and Spectral Structure in the X-Ray Emission from the Crab Nebula. Astrophys. J. 536, L81-L84 (2000).

7. Khangoulian, D. V. \& Bogovalov, S. V. The Role of a Magnetic Field in the Formation of Jet-like Features in the Crab Nebula. Astron. Lett. 29, 495-501 (2003).

8. Del Zanna, L., Amato, E. \& Bucciantini, N. Axially symmetric relativistic MHD simulations of Pulsar Wind Nebulae in Supernova Remnants. On the origin of torus and jet-like features. Astron. Astrophys. 421, 1063-1073 (2004).

9. Komissarov, S. S. \& Lyubarsky, Y. E. Synchrotron nebulae created by anisotropic magnetized pulsar winds. Mon. Not. R. Astron. Soc. 349, 779-792 (2004).

10. Bogovalov, S. V., Chechetkin, V. M., Koldoba, A. V. \& Ustyugova, G. V. Interaction of pulsar winds with interstellar medium: numerical simulation. Mon. Not. R. Astron. Soc. 358, 705-715 (2005).

11. Porth, O., Komissarov, S. S. \& Keppens, R. Three-dimensional magnetohydrodynamic simulations of the Crab nebula. Mon. Not. R. Astron. Soc. 438, 278-306 (Feb. 2014).

12. Peierls, R. Model-Making in Physics. Contemp. Phys. 21, 3 (1980).

13. Aharonian, F. A. \& Atoyan, A. M. Compton scattering of relativistic electrons in compact X-ray sources. Astrophys. Space Sci. 79, 321-336 (1981). 